\title{
COMPLETE MINIMAL SURFACES AND THE PUNCTURE NUMBER PROBLEM
}

\author{
KICHOON YANG
}

(Communicated by Christopher Croke)

\begin{abstract}
Given a nonnegative integer $g$, let $\mathscr{P}(g)$ denote the set of integers $N$ such that an arbitrary compact Riemann surface with genus $g$ can be completely conformally and minimally immersed in $\mathbb{R}^{3}$ (with finite total curvature) with exactly $N$ punctures. We prove that the infimum of $\mathscr{P}(g)$ is at most $4 g$ and that the set $\mathscr{P}(g)$ may not miss any $3 g$ consecutive integers larger than the infimum of $\mathscr{P}(g)$.
\end{abstract}

\section{INTRODUCTION}

A conformal immersion from a Riemann surface $M$ to $\mathbb{R}^{3}$ is said to be minimal if its component functions are harmonic, and the maximum principle for harmonic functions prohibits any compact minimal surface. The tangential Gauss map of a conformal minimal immersion $f: M \rightarrow \mathbb{R}^{3}$ is the map $\Phi: M \rightarrow G(3,2)$ taking $p \in M$ to the (negatively) oriented tangent plane $f_{*} T_{p} M \subset \mathbb{R}^{3} . G(3,2)$ is the Grassmann manifold of oriented two-planes in $\mathbb{R}^{3}$, and it lies naturally in $\mathbb{C} P^{2}$. Moreover, the minimality of $f$ implies that the map $\Phi: M \rightarrow \mathbb{C} P^{2}$ is holomorphic. A fundamental theorem of Chern and Osserman $[\mathrm{CO}]$ states that a complete minimal surface $M$ (relative to the induced metric) is of finite total Gaussian curvature if and only if $M$ is holomorphically equivalent to a compact Riemann surface $M_{g}$ punctured at a finite number of points and the tangential Gauss map extends holomorphically to all of $M_{g}$. Thus the theory of complete minimal surfaces of finite total curvature is intimately linked to that of compact Riemann surfaces.

Given a nonnegative integer $g$, define the set $\mathscr{P}(g) \subset \mathbb{Z}^{+}$by the following prescription: $N \in \mathscr{P}(g)$ if and only if any compact Riemann surface of genus $g$ can be completely conformally and minimally immersed in $\mathbb{R}^{3}$ (with finite total curvature) with exactly $N$ punctures. In less precise terms, the set $\mathscr{P}(g)$ consists of admissible puncture numbers for an arbitrary compact Riemann surface of genus $g$. For example, it is well known [JM] that $\mathscr{P}(0)=\mathbb{Z}^{+}$. Hereafter we will exclude the case $g=0$ from our consideration. We can now state the

Puncture number problem. Determine the set $\mathscr{P}(g), g>0$.

Received by the editors February 3, 1992.

1991 Mathematics Subject Classification. Primary 53A10. 
Our main result is the following

Theorem. (a) $4 \in \mathscr{P}(1)$,

(b) $\inf \mathscr{P}(g) \leq 4 g$,

(c) $\mathscr{P}(g)$ may not miss any $3 g$ consecutive integers larger than inf $\mathscr{P}(g)$.

Note that (c) implies that $|\mathscr{P}(g)|=\infty$. That is to say, there are infinitely many admissible puncture numbers for any genus.

It should be remarked that the puncture number problem is closely related to the moduli problem for complete minimal surfaces. In fact, Xiaokang Mo recently constructed a complex space parametrizing the set of complete minimal surfaces with a fixed number of punctures using an earlier result of the author [Y1] on the puncture number problem. For details of Mo's construction see [M].

\section{AN eStiMATE LEMMA AND THE PROOF OF THE THEOREM}

To prove the Theorem we will need the following

Lemma. Let $M_{g}$ denote a compact Riemann surface of genus $g>0$. Also let $F \in H^{0}\left(M_{g}, \mathscr{M}^{*}\right)$ be a not identically zero meromorphic function on $M_{g}$, and put

$$
\begin{aligned}
n & =n_{F}=\text { the number of distinct poles of } F \\
d & =d_{F}=\text { the degree of } F=\text { the total number of poles of } F, \\
m & =m_{F}=\text { the number of distinct zeros of } d F
\end{aligned}
$$

(i) There exists a conformal minimal immersion, complete with respect to the induced metric, $M_{g} \backslash \Sigma_{F} \rightarrow \mathbb{R}^{3}$, where the puncture set $\Sigma_{F}$ satisfies

$$
n+m \leq\left|\Sigma_{F}\right| \leq 2 n+d+3 g-2 .
$$

(ii) Suppose $D$ is any divisor on $M_{g}$ satisfying the following conditions:

$$
\begin{aligned}
\operatorname{deg} D=- & g, \quad D^{+}=(d F)_{0}, \quad D^{-} \geq(d F)_{\infty}, \\
\operatorname{support} D^{-} & =\operatorname{support}(d F)_{\infty},
\end{aligned}
$$

where $D=D^{+}-D^{-}$with $D^{ \pm}>0$. Also let $G \in L(-D)$, and put

$$
m^{\prime}=\text { the number of distinct zeros of } G \text {. }
$$

Then there exists a complete conformal minimal immersion $M_{g} \backslash \Sigma_{F, G} \rightarrow \mathbb{R}^{3}$ with

$$
\left|\Sigma_{F, G}\right|=n+m^{\prime}
$$

Proof. For some $b_{i} \in \mathbb{Z}^{+}$and distinct points $p_{i} \in M_{g}$ we have

$$
(F)_{\infty}=\sum_{i=1}^{n} b_{i} p_{i}
$$

Then $d=\sum b_{i}=\operatorname{deg}(F)_{\infty}$. Consider the meromorphic 1-form $d F$. We have

$$
(d F)_{\infty}=\sum\left(b_{i}+1\right) p_{i}
$$


For some $a_{j} \in \mathbb{Z}^{+}$and distinct points $q_{j} \in M_{g}$ we have

$$
(d F)_{0}=\sum_{j=1}^{m} a_{j} q_{j} .
$$

Since $(d F)=(d F)_{0}-(d F)_{\infty}$ is a canonical divisor, its degree is $2 g-2$ so that

$$
\sum a_{j}=(2 g-2)+n+d .
$$

Let $D=D^{+}-D^{-} \in \operatorname{Div}\left(M_{g}\right)$ be as above. We then have

$$
D^{+}=\sum_{j=1}^{m} a_{j} q_{j}, \quad D^{-}=\sum_{i=1}^{n} c_{i} p_{i},
$$

where the $c_{i}$ 's are some positive integers satisfying the conditions

$$
c_{i} \geq b_{i}+1 ; \quad \sum c_{i}=3 g-2+n+d .
$$

Consider the complex vector space

$$
L(-D)=\left\{\varphi \in H^{0}\left(M_{g}, \mathscr{M}^{*}\right):(\varphi) \geq D\right\} \cup\{0\} .
$$

Since the support of $D^{+}=(d F)_{0}$ is nonempty, we note that nonzero constant functions may not lie in $L(-D)$. By the Riemann-Roch theorem

$$
\begin{aligned}
\operatorname{dim} L(-D) & =\operatorname{deg}(-D)-g+1+\operatorname{dim} L((d F)+D) \\
& =1+\operatorname{dim} L((d F)+D) \geq 1 .
\end{aligned}
$$

Thus there are nonconstant meromorphic functions belonging to $L(-D)$. Let $G$ be such a function, and also let $m^{\prime}$ denote the number of distinct zeros of $G$. Using an argument totally similar to the one given in [Y1, pp. 708-710] we can find a complete conformal minimal immersion $M_{g} \backslash \Sigma \rightarrow \mathbb{R}^{3}$, where

$$
\Sigma=\operatorname{support}(F)_{\infty} \cup \operatorname{support}(G)_{0} .
$$

But $F$ has $n$ distinct poles and $G$ has $m^{\prime}$ distinct zeros. This establishes the equality in $(* *)$. Since $(G)_{0} \geq D^{+}=\sum_{j=1}^{m} a_{j} q_{j}$, we must have $m^{\prime} \geq m$, proving the first inequality in $(*)$. On the other hand, $m^{\prime}$ is at most equal to the degree of $D^{-}$: the degree of $D$ is negative and $G$ can have at most $\operatorname{deg}\left(D^{-}\right)$many simple zeros. So

$$
m^{\prime} \leq \sum c_{i}=3 g-2+n+d .
$$

The rest follows easily.

Proof of Theorem. We first consider the case $g=1$. Let $M_{1}$ be any complex torus, and also let $\mathfrak{p}(z)$ denote the Weierstrass function on it, where $z$ is the (global) Euclidean coordinate on $M_{1}$. Let $p \in M_{1}$ be the lattice point so that

$$
(\mathfrak{p})_{\infty}=2 p \text {. }
$$

Now $d \mathfrak{p}=\mathfrak{p}^{\prime} d z$, and it is well known that $\mathfrak{p}^{\prime}$ has three distinct simple zeros, say $q_{1}, q_{2}$, and $q_{3}$. Thus

$$
(d \mathfrak{p})=\left(q_{1}+q_{2}+q_{3}\right)-3 p .
$$


Taking $F=\mathfrak{p}$ in the Lemma, we find that $d=2, n=1, m=3$. Now take

$$
D=\left(q_{1}+q_{2}+q_{3}\right)-4 p .
$$

Then $G=\mathfrak{p}^{\prime} \in L(-D)$ and $m^{\prime}=3$. It follows that $\left|\Sigma_{F, G}\right|=4$, proving (a) of the Theorem. We now assume that $g>1$. We will show that we can pick $F \in H^{0}\left(M_{g}, \mathscr{M}^{*}\right)$ so that

$$
2 n_{F}+d_{F}+3 g-2 \leq 4 g .
$$

Then the last inequality in $(*)$ will give (b) of the Theorem. Let $p \in M_{g}$ be any Weierstrass point. This means that the gap sequence at $p$ is not $\{1,2, \ldots, g\}$. In particular, there is a nongap $\tilde{d} \leq g$ at the point $p$, meaning that there is a meromorphic function $\widetilde{F}$ on $M_{g}$ with $(\widetilde{F})_{\infty}=\tilde{d} p$. Take $F=\widetilde{F}$. Then $d=\tilde{d}, n=1$, and ( $\dagger$ ) follows. Before proving (c) in general we first look at the case $g=1$. We have the following

Observation. Let $M_{1}$ be any complex torus, and also let $\tilde{n} \geq 2$ be an integer. Then we can find a principal divisor on $M_{1}$ of the form

$$
\sum p_{i}-\sum q_{i}, \quad 1 \leq i \leq \tilde{n},
$$

where the $p_{i}$ 's and the $q_{i}$ 's are distinct points of $M_{1}$.

The above observation is proved easily from Abel's theorem, which in this case states that a degree zero divisor on $M_{1}$ is principal if and only if the group sum (in the abelian group $M_{1}$ ) of the points of $D$ is zero.

Given $M_{1}$ and any $n=\tilde{n} \geq 2$, we take $F$ such that

$$
(F)=\sum_{i=1}^{n}\left(p_{i}-q_{i}\right), \quad \text { the } p_{i} \text { 's and } q_{i} \text { 's are distinct. }
$$

Then $(*)$ gives

$$
n+1 \leq\left|\Sigma_{F}\right| \leq 3 n+1
$$

since $d=n=\tilde{n}$ and $m \geq 1$. On the other hand, we can take $F$ to be such that $(F)_{\infty}=d p$, where $p$ is any point of $M_{1}$ and $d$ is any integer $\geq 2$. Then $n=1$, and the Lemma gives

$$
2 \leq\left|\Sigma_{F}\right| \leq d+3 .
$$

The inequalities in (1), (2) prove (c) for the case $g=1$. Now let $M_{g}$ denote a Riemann surface of genus $g>1$. Then, given any $d>g$, there is a meromorphic function $F$ with $(F)_{\infty}=d p, p \in M_{g}$; just take $p$ to be any non-Weierstrass point. Then $n=1$ and the Lemma gives

$$
\left|\Sigma_{F}\right| \leq 3 g+d .
$$

On the other hand, given any $n \in \mathbb{Z}^{+}$, there is a meromorphic function $F$ on $M_{g}$ with $n$ distinct poles (just add $n$ "gap functions" at $n$ distinct points). So given any $n \in \mathbb{Z}^{+}$we can have

$$
n+1 \leq\left|\Sigma_{F}\right| .
$$

Combining (3), (4) we obtain the following 
Proposition. Given any Riemann surface $M_{g}$ of genus $g$ and any integer $d>$ $g$, there exists a complete conformal minimal immersion $M_{g} \backslash \Sigma \rightarrow \mathbb{R}^{3}$ with

$$
d+1 \leq|\Sigma| \leq 3 g+d .
$$

The above proposition easily implies (c) of the Theorem.

\section{CONCLUDING REMARKS}

One suspects that $\mathscr{P}(g) \supset\{N \in \mathbb{Z}: N \geq 4 g\}$-a sort of deformation argument might work. On the other hand, it is unclear that $4 g$ is actually the minimum of $\mathscr{P}(g)$. For example, one can reduce the degree of the meromorphic function used in producing the lower bound $4 g$ : on any $M_{g}$ it is well known that there is a meromorphic function of degree $d \leq(g+3) / 2$. However, it seems difficult to determine the number of distinct poles of such a meromorphic function.

\section{ACKNOWLEDGMENT}

Finally, the author would like to acknowledge several interesting conversations with Xiaokang Mo and is grateful to Bob Osserman for inspiration and encouragement.

\section{REFERENCES}

[B] D. Bloss, Elliptische funktionen und vollstandige minimalflachen, doctoral dissertation, Freien Universitat, Berlin, 1989.

[CO] S. Chern and R. Osserman, Complete minimal surfaces in Euclidean $n$-space, J. Analyse Math. 19 (1967), 15-34.

[GK] F. Gackstatter and R. Kunert, Konstruktion vollstänger Minimalflächen von endlicher Gesamtkrümmung, Arch. Rational Mech. Anal. 65 (1977), 289-297.

[JM] L. Jorge and W. Meeks, The topology of complete minimal surfaces of finite total Gaussian curvature, Topology 22 (1983), 203-221.

[KS] T. Klotz and L. Sario, Existence of complete minimal surfaces of arbitrary connectivity and genus, Proc. Nat. Acad. Sci. U.S.A. 54 (1965), 42-44.

[M] X. Mo, Gauss map and moduli space of minimal surfaces in Euclidean spaces, Doctoral dissertation, Stanford University, Stanford, CA, 1990.

[O] R. Osserman, A survey of minimal surfaces, Van Nostrand, New York, 1969.

[Y1] K. Yang, Meromorphic functions on a compact Riemann surface and associated complete minimal surfaces, Proc. Amer. Math. Soc. 105 (1989), 706-711.

[Y2] K. Yang, Complete and compact minimal surfaces, Kluwer Academic Publishers, Boston. MA, 1989.

Department of Mathematics, Arkansas State University, State University, Arkansas

E-mail address: yang@quapaw.astate.edu 